\title{
The Dynamo Project: The World's First Robot Soccer Players
}

\author{
Alan K. Mackworth*1
}

\begin{abstract}
This Dynamo project overview briefly describes the development of the world's first autonomous robot soccer players. The accompanying video shows the outcomes of a series of experiments using small scale radio-controlled trucks and cars to play robot soccer. These pioneering experiments in embodied intelligence were successfully carried out over the period 1992-1994 in the Dynamo project at the UBC Laboratory for Computational Intelligence. They served as precursors to the RoboCup robot soccer competitions that started in 1997.
\end{abstract}

\section{INTRODUCTION}

In 1992, the goal of creating an integrated cognitive robot was still only a tantalizing dream. Artificial intelligence and robotics research was highly divergent with little or no commonality among specialized subfields. New rich task domains were needed to pose the right challenges to extant theories and robot prototypes and promote convergence. In [1], we proposed soccer playing as such a task since it requires situated robotics, perception, real-time decision making, planning, plan recognition, learning and multi-robot coordination and control.

At that time, the technology to perform real-time vision and build autonomous robots with off-the-shelf hardware was just becoming available. In our Dynamo (DYNAmics and MObile robots) project we built two testbeds. The first prototype used radio-controlled (R/C) 1/12 scale model monster trucks (Clodbusters) shown in [1]. The second prototype, the Dynamite testbed, used 1/24 scale R/C cars. The Dynamite testbed was built to perform experiments with multiple robots. Soccer tournaments were carried out using the testbed to evaluate aspects of the proposed reactive deliberation robot architecture. The results raised new issues and problems for research on robotic agents operating in dynamic environments and led to the development of the RoboCup soccer tournaments.

The Good Old Fashioned AI and Robotics (GOFAIR) [1] research paradigm had shaped the discipline of robotics since its early days. Some of the fundamental assumptions made about the world in GOFAIR were that there is only one agent, that the environment is static unless the agent changes it, that actions are discrete and are carried out sequentially and that the world the robot inhabits can be accurately and exhaustively modeled by the robot. These assumptions proved to be overly restrictive and ultimately sterile. In the

*This work was supported by the Natural Sciences and Engineering Research Council, the IRIS Network of Centres of Excellence and the Canadian Institute for Advanced Research.

${ }^{1}$ Alan K. Mackworth holds the Canada Research Chair in Artificial Intelligence in the Department of Computer Science, University of British Columbia, Vancouver, BC, Canada. mack@cs.ubc.ca, wWw. cs. ubc. ca/ mack usual dynamic of the scientific dialectic, a new movement emerged as the antithesis to GOFAIR: the Situated Agent approach. The Situated Agent paradigm is characterized by the guiding principles set forth by Brooks [2], Mackworth [1,3], Pfeifer [4] and others: intelligence should be embodied, embedded and emergent. The key idea of situatedness and embodiment is that researchers should consider embodied agents that interact with a larger world that provides the context for their activity. The essence of intelligence and emergence is that the intelligence of an agent can be judged by the quality of its interaction with its environment.

\section{WHY ROBOT SOCCER AS A TASK DOMAIN?}

We proposed that playing soccer be a paradigmatic task domain since it breaks with nearly all of the restrictive assumptions on which GOFAIR is based and meets the standards proposed in the Situated Agent approach [3]. The soccer domain has the following characteristics:

- Neutral, friendly, and hostile agents

- Interagent cooperation

- Real-time interaction

- Dynamic environment

- Real and unpredictable world

- Objective performance criteria

- Repeatable experiments

The GOFAIR assumptions do not hold in the soccer world. The one agent assumption is violated: there are cooperating agents on the robots team, competing agents on the other team, and neutral agents such as the referee and the weather. The world is not completely predictable: it is not possible to predict precisely where the ball will go when it is kicked, even if all the relevant factors are known. The simplifying assumption of discrete sequential actions is violated: continuous events such as a player running to a position and the ball moving through the air occur concurrently.

In soccer, robot agents are embodied and are situated in an unfolding game. Although it is still true that the intelligence of an agent can be judged from the dynamics of interaction with the environment, soccer also provides objective performance criteria.

The ability to score and prevent goals and the overall score of the game are objective measures of success. These measures allow explicit comparisons of alternative controller designs. The effects of chance can be factored out by carrying out repeated experiments. With objective criteria and repeatability, short-term and long-term learning strategies, as well as experiments in automatic evolution of controllers, become feasible. The availability of objective criteria is a critical feature of soccer that distinguishes it, along with the 
aspect of a real and unpredictable environment, from many of the other task domains proposed for driving the situated agent research paradigm.

Soccer as a task domain is sufficiently rich to support research integrated from many branches of AI and Robotics. In addition to the obvious potential of the soccer domain for research in perception and motor control, there are many other areas of AI that are applicable: reasoning under uncertainty, on-line reasoning, resource-bounded reasoning, planning, decision theory, qualitative physics, plan recognition, learning, and multi-agent theory.

Soccer is not the real world, but a suitably circumscribed fragment of it. Soccer is an appropriate abstraction of the world that challenged research in Robotics and AI to focus on achievable tasks, and to drive the development of relevant theories.

\section{DYNAMITE: A TESTBED FOR MULTIPLE MOBILE ROBOTS}

The Dynamite testbed provided a practical platform for testing theories in the soccer domain using multiple mobile robots. The testbed consisted of a fleet of radio controlled vehicles that perceive the world through a shared perceptual system [5,6]. In an integrated off board environment with dataflow and MIMD computers, vision programs monitored the position and orientation of each robot while planning and control programs generated and sent out motor commands. This approach allowed umbilical-free behaviour and very rapid, lightweight fully autonomous robots.

The mobile robot bases were commercially available radio controlled vehicles. We had four controllable 1/24 scale racing-cars, each $22 \mathrm{~cm}$ long, $8 \mathrm{~cm}$ wide, and $4 \mathrm{~cm}$ high excluding the antenna. The testbed was $244 \mathrm{~cm}$ by $122 \mathrm{~cm}$. The cars were each fitted with two circular colour markers to allow the vision system to identify their position and orientation. There was a single colour camera mounted in a fixed position above the soccer field. The video output of the camera was transmitted to special-purpose video processing DataCube hardware. The DataCube was a dataflow computer which was programmed to classify image pixels into different colour classes at video rate $(60 \mathrm{~Hz})$. This information was transmitted to a network of transputers which formed a MIMD computer. Additional vision processing was performed on the transputers to find the position, in screen coordinates, of the centroid of each coloured blob and to transform these positions from screen to world coordinates. The vision subsystem was called the Vision Engine [5]. The Vision Engine produced the absolute position of all the objects on the soccer field; the orientation of each car is also reported. This was done at $60 \mathrm{~Hz}$ with an accuracy in position of approximately $1 \mathrm{~mm}$.

The reasoning and control components of a vehicle could be implemented on any number of transputers out of the available pool. Each vehicle was actually controlled by a distributed user program running on two transputer nodes. An arbitrary number of nodes could be used in parallel to control independent vehicles. The movement of all vehicles was controlled through radio transmitters attached to a single shared transputer node. Commands were transmitted to the vehicles at a rate of $50 \mathrm{~Hz}$. As described in [6,7], we experimented with various control regimes that combined reactive behaviours and deliberative planning, and ran tournaments to determine the best architectures and behaviours.

\section{THE DYNAMO PROJECT VIDEO}

The accompanying video has six segments:

1) Introduction with title slide

2) Equipment: the experimental setups

3) A Tale of Two Monster Trucks: two Clodbusters playing soccer (1992)

4) A Tale of Two Dynamites: one-on-one soccer with two cars (1993)

5) A Tale of Four Dynamites: two-on-two soccer with four cars (1994)

6) Reprise: conclusion

\section{CONCLUSION}

These Dynamo project experiments, and the ideas behind them, were the first in a series of robot soccer playing robots that led to the establishment of the immensely successful RoboCup [8], with the first RoboCup tournament held in 1997. The RoboCup Small League still uses the overhead global vision system developed in the Dynamo project. In the Small League the robots are now custom, sophisticated, expensive and holonomic as opposed to the off-the-shelf, cheap non-holonomic cars and trucks used in the Dynamo project. In the RoboCup Middle Size and Simulation Leagues local, situated vision systems are used.

\section{ACKNOWLEDGMENTS}

Some of the material in this project overview is abstracted and summarized from papers [1,3,5,6]. Rod Barman, Keiji Kanazawa, Stewart Kingdon, Jim Little, Dinesh Pai, Michael Sahota, Heath Wilkinson and Ying Zhang collaborated on the Dynamo robot soccer project. Pooyan Fazli edited and produced the video.

\section{REFERENCES}

[1] A. K. Mackworth, "On Seeing Robots," in Computer Vision: Systems, Theory and Applications, A. Basu and X. Li, Eds., World Scientific Press, Singapore, 1993, pp. 1-13. (Presented at Vision Interface 92 (VI-92), Vancouver, BC, May 1992.)

[2] R. A. Brooks, "Elephants Don't Play Chess" Robotics and Autonomous Systems, vol. 6, pp. 3-15, 1990.

[3] A. K. Mackworth, "Agents, Bodies, Constraints, Dynamics, and Evolution," AI Magazine, vol. 26, no. 1, pp. 7-28, Spring, 2009.

[4] R. Pfeifer and C. Scheier, Understanding Intelligence. Cambridge, MA:MIT Press, 1999.

[5] A. R. Barman, S. J. Kingdon, A. K. Mackworth, D. K. Pai, M. K. Sahota, H. Wilkinson, and Y. Zhang, "Dynamite: A Testbed for Multiple Mobile Robots," In Proc. IJCAI Workshop on Dynamically Interacting Robots, Chambery, France, 1993, pp. 38-45.

[6] M. Sahota and A. K. Mackworth, "Can Situated Robots Play Soccer?" in Proc. Artificial Intelligence 94, Banff, AB, Canada, 1994, pp. 249254.

[7] M. K. Sahota. "Reactive deliberation: An architecture for real-time intelligent control in dynamic environments," in Proc. of the Twelfth National Conference on Artificial Intelligence (AAAI-94), Seattle, WA, 1994, pp. 1303-1308,

[8] http://www.robocup.org/ 